\title{
Transfer Learning Based Multiclass Classification For Covid-19 Detection Using Chest X-Rays
}

Japman Singh Monga ( $\nabla$ japman310@gmail.com )

National Institute Of Technology Delhi

Yuvraj Singh Champawat ( $\nabla$ yuvrajsingh19082000@gmail.com )

National Institute Of Technology Delhi

Dr. Seema Kharb ( $\nabla$ seema016@gmail.com )

SRM University, Delhi-NCR, Sonipat

\section{Research Article}

Keywords: Corona virus (Covid-19), Transfer Learning, Multi-Class Classification, Pneumonia, Chest X-Ray Images, Deep Learning

Posted Date: October 11th, 2021

DOI: https://doi.org/10.21203/rs.3.rs-810768/v2

License: (9) This work is licensed under a Creative Commons Attribution 4.0 International License.

Read Full License 


\title{
Transfer Learning Based Multiclass Classification For Covid-19 Detection Using Chest X-Rays
}

\author{
Japman Singh Monga ${ }^{1 *}$ \\ ,Yuvraj Singh Champawat ${ }^{2 *}$ and Dr. Seema Kharb ${ }^{3 *}$ \\ 1,2,3 National Institute of Technology Delhi, Delhi , India \\ \{japman310, yuvrajsingh19082000, seema016\}@gmail.com
}

\begin{abstract}
In the year 2020 world came to a halt due to spread of Covid-19 or SARS-CoV2 which was first identified in Wuhan, China. Since then, it has caused plethora of problems around the globe such as loss of millions of lives, economic instability etc. Less effectiveness of detection through RT-PCR and also prolonged time needed for detection through RT-PCR calls for a substitute for Covid-19 detection. Hence, in this study, we aim to develop a transfer learning based multi-class classifier using Chest X-Ray images which will classify the XRay images in 3 classes (Covid-19, Pneumonia, Normal). Further, the proposed model has been trained with deep learning classifiers namely: DenseNet201, Xception, ResNet50V2, VGG16, VGG-19, InceptionResNetV2 .These are evaluated on the basis of accuracy, precision and recall as performance parameters . It has been observed that DenseNet201 is the best deep learning model with $82.2 \%$ accuracy.
\end{abstract}

Keywords: Corona virus (Covid-19), Transfer Learning, Multi-Class Classification, Pneumonia, Chest X-Ray Images, Deep Learning

\section{Introduction}

In the year 2020 world came to a halt due to spread of a virus from the family of Corona Virus and was named as Covid-19 or SARS-CoV2. Covid-19 was first identified in Wuhan, China in December 2019 and since then, it has spread to almost every country of the world ,hence being declared as a pandemic[1]. More than 2 million people worldwide have already succumbed to death due to the Covid-19[1], making it one of the deadliest pandemics ever encountered.

Corona viruses result in illnesses such as respiratory and gastrointestinal diseases[2]. Covid19 is a Severe Acute Respiratory Syndrome (SARS) and is highly contagious[2]. Moving on to the symptoms of the COVID-19, for those who start to develop the symptoms, the symptoms might be very moderate as that of a seasonal flu[3]. Incubation period of the virus is 2 to 14 days[4] which is longer than other viruses making it highly risky. The common symptoms of the virus include fever, tiredness, cough, loss of taste, muscle ache, fatigue, sore throat[4]. It is 
worthy to note that for the people who show severe symptoms, the symptoms are that of pneumonia as it mainly affects lungs.

Unavailability of the vaccine for the virus and high mortality rate has resulted in the collapse of medical facilities in many countries of the world. The standard procedure to detect the virus is Reverse Transcription Polymerase Chain Reaction (RT-PCR)[3], the test kits of which confirm the virus by gene sequencing for respiratory or blood samples as a key indicator. However, the current data asserts that RT-PCR is only 30\%-70\% effective for acute infection[5]. The time taken for the results to come through RT-PCR is supposed to be 6 to 8 hours. However, the availability and testing time varies from country to country, region to region which has resulted in slow detection of the virus and has eventually resulted in more people getting affected.

This calls for finding an alternative automated method for detection of Covid-19. Imaging is viewed as a potential alternative solution due to high availability of imaging technologies in many countries. Among the imaging technologies, Chest X-Ray and Chest CT Scans have been regarded as the most potential methods of detection[6]. However, main disadvantage of using CT imaging is high cost[6]. Therefore, this paper focuses only on X Ray imaging modality for detection. Various techniques have been invented for diagnosis of diseases through imaging. Several traditional ML techniques have proved very beneficial for regression, classification and unsupervised analysis[7]. ANN, which are composed of artificial neurons have had very good performance for these tasks but also had many limitations such as decrease in the local minimum during optimization, and over-training for given values (overfitting)[7]. To counter this, deep learning architectures have been used to determine complex features and relationships.

In the past years, Deep Learning has proved to be efficient in determining complex relationships in the data and has given astonishing results. Convolutional Neural Networks, or CNNs, have proved to be very efficient for image recognition and classification tasks and have been used in many medical tasks[8]. But as of now, data available in open source for Covid-19 is very less, hence transfer learning has proved to be beneficial. Transfer Learning is a method that allows us to use knowledge gained from other tasks in order tackle new but similar problems quickly and effectively[9], hence we could use the weights of pre-trained models that were trained on a huge dataset of images (Image Net). So, transfer learning is a viable method that can be applied in this detection framework. Also, as discussed, Covid-19 shows symptoms of pneumonia, hence it's need of the hour to build a classifier which not only classifies Covid-19 and normal patients but also differentiates between Covid-19 and pneumonia patients. Hence, the aim of this paper is to propose a transfer learning-based mechanism to perform multi-class classification between the 3 classes (Normal, Pneumonia, Covid-19) and try to find out the most appropriate network among various deep learning networks. This paper will aid researchers to develop a system to detect Covid-19 using the vast technology stack available in today's world.

The rest of the paper has following sections. Section 2 gives overview of related research work that has been done in regard to this area. Methodology proposed and used along with description of various deep learning classifiers have been given in section 3. Section 4 contains all the implementation details related to study. Section 5 comprises of all the empirical results obtained, their analysis and comparative performance. To sum up, the last section provides the summary of the paper and discusses the future scope of this study. 


\section{Literature Review}

Various research studies have been done around the globe for Covid-19 detection. In most of the studies, deep learning techniques are employed on chest radiography images with an aim to detect infected patients. The results obtained from these studies have been promising keeping accuracy as a metric. Table 1 summarizes the key features of these studies

Table 1. Key Features of related studies.

\begin{tabular}{|c|c|}
\hline Title & Advantages \\
\hline $\begin{array}{l}\text { Ezz El-Din Hemdan, } \\
\text { Marwa A. Shouman , } \\
\text { and Mohamed Esmail } \\
\text { Karar , IEEE, } \\
\text { Member [6] }\end{array}$ & $\begin{array}{l}\text { Pre-defined CNN networks are fine-tuned on } \\
\text { CXR dataset for } 2 \text { class classification and their } \\
\text { performances are compared. } \\
\text { Apart from accuracy, training time is also } \\
\text { compared }\end{array}$ \\
\hline
\end{tabular}
Disadvantages

Major drawback of this paper is overfitting. It is seen that these pre-trained

Pre-defined CNN networks are fine-tuned on networks fine-tuned on CXR images dataset shows over-fitting compared

due to which their training accuracy is high but the test accuracy is fairly low for most of network models.

CNN models were fine-tuned to identify and Antonios Makris, classify the different classes (Covid-19, Ioannis Kontopoulos pneumonia, normal). The key point of this and Konstantinos paper was the high specificity in case of Tserpes [10] COVID-19 images classification which is must for diagnosis.

Major drawback of this paper was also overfitting in some models

The training set used

Covid Net, a new architecture is introduced in this paper. Main advantage is no patient overlap between the test and the training set,

L. Wang, A. Wong, [11] which is very important in these kinds of problems.

in this paper is highly imbalanced with 183 cases of COVID-19, 5,538 of Pneumonia and 8,066 of normal healthy subjects

I. Castiglioni, D. Ippolito, $\mathrm{M}$. Interlenghi, C.B. Monti, C. Salvatore, S. Schiaffino, A. Polidori, D. Gandola, C. Messa, F. Sardanelli, [12]

The authors proposed a completely different protocol using a dataset that they collected and that is not public. They trained an ensemble of 10 ResNets.

They used both anteroposterior and posteroanterior projections and they do not suffer of the dataset recognition problem

The author presented a novel architecture based on fine tuning of pre-trained networks

Ali Narin, Ceren Kaya, Ziynet Pamuk, [13]
The model achieved a ROC-AUC of 0.80 for the classification task. Its performance is much than the other ones mentioned.

The major drawback of this paper is a very small dataset consisting of only 100 images ( 50 of COVID19 and 50 of Normal) like Inception-V3, ResNet50 and Inception

ResnetV2. . To avoid overfitting in the models $\mathrm{k}$-fold cross validation is applied and accuracies of models in each fold of validation 
Apart from the above mentioned studies, DarkNet model [14] been one of the most comprehensive study topical to this area which provides both binary and multiclass classification and proposes a DarkNet model[14]. From the above table, it can be noted that main disadvantages of most studies have been overfitting, class imbalance or small dataset. In this paper, we try to counter these disadvantages using various techniques.

\section{Proposed Methodology}

Before describing the methodology, a major hindrance in the study has to be noted. The amount of dataset available for the problem statement is fairly low. So, a deep convolutional network cannot be trained from scratch using this small dataset. Hence, in the proposed model, the concept of transfer learning has been employed. Transfer Learning is a method that allows us to use knowledge gained from other tasks in order tackle new but similar problems quickly and effectively, weights of pre-trained models that were trained on a huge dataset of images (Image Net) can be used [9].The proposed model comprises of two stages, as shown in Fig 1:

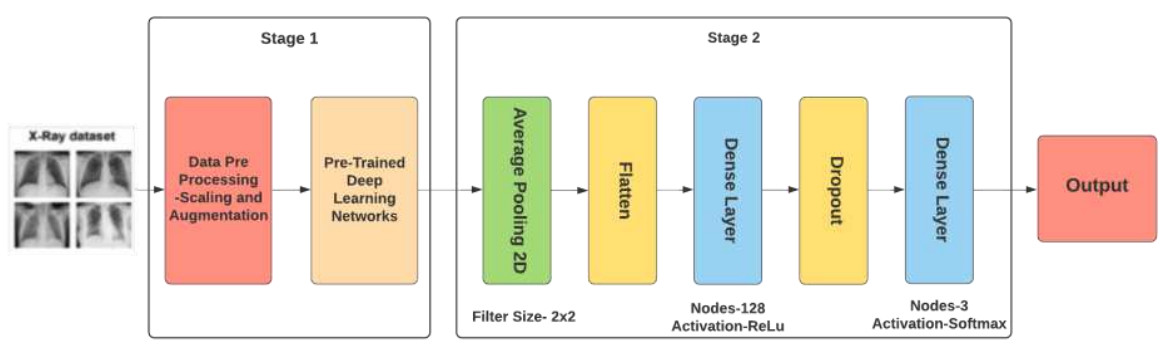

Fig. 1. The proposed model in the study.

\subsection{Stage 1- Data Preprocessing and Feature Extractor}

\section{Data Preprocessing}

Since all the images might be derived from the variegated sources, so all the images are scaled to a common size of $224 \times 224$. Also, rotation of 15 degrees has been applied as a part of data augmentation. After data preprocessing phase, the images serve as an input to the model.

\section{Pre-Trained Deep Learning Networks (Feature Extractor)}

In order to train the model pre-trained deep learning models have been used. Here, transfer learning has been used and this stage acts as feature extractor for the proposed model. Also, the weights of pre-trained models are non-trainable, therefore these remain unchanged during training. The top layers of these pre-trained models have been excluded, so the output from this stage serves as input for the next stage. The classifier is built on top of this model and has been discussed in next sub section. The pre-trained deep learning models which are used are as follows: 
$V G G 16$ and $V G G 19$

Visual Geometry Group Network (VGG) was developed based on the convolutional neural network architecture by Oxford Robotics Institute's Karen Simonyan and Andrew Zisserman[15].VGG networks, instead of having a large number of hyper-parameter focused on having convolution layers of $3 \times 3$ filter with a stride 1 and always used same padding and maxpool layer of $2 \times 2$ filter of stride 2 . It follows the arrangement of convolution and max pool layers consistently throughout the whole architecture[15]. VGG 16 and VGG 19 are two available versions of this network and they differ only in terms of depth and number of layers with 16 and 19 being the number of layers in the networks.

\section{DenseNet 201}

DenseNets are divided into DenseBlocks, where the dimensions of the feature maps remains constant within a block, but the number of filters changes between them[16]. These layers between them are called Transition Layers and take care of the down sampling applying a batch normalization, a 1x1 convolution and a $2 \times 2$ pooling layers. Dense nets have proved to be very beneficial in some of the aspects such as- the number of parameters reduce substantially, vanishing gradient problem is addressed, and it encourages feature reuse[16].

\section{Xception}

The Xception architecture has 36 convolutional layers forming the feature extraction base of the network[17].Convolutional base is be followed by a logistic regression layer. Optionally one may insert fully-connected layers before the logistic regression layer. The 36 convolutional layers are structured into 14 modules, all of which have linear residual connections around them, except for the first and last modules.[17]

Inception ResNet V2

This convolutional neural network is 164 layers deep, combining the Inception architecture with residual connections [18]. Inception-ResNet-V2 is a variation of InceptionV3. InceptionResNet$\mathrm{V} 2$ is trained on more than a million images from the ImageNet database.[18]

\section{$\operatorname{ResNet50~V2~}$}

ResNet50 version 2 uses the pre-activation of weight layers instead of post-activation[19]. ResNet50 V2 has removed the last non-linearity, therefore, clearing the path of the input to output in the form of identity connection .ResNet V2 applies Batch Normalization and ReLU activation to the input before the multiplication with the weight matrix (convolution operation)[19].

\subsection{Stage 2- Fine Tuning and Classifier Stage}

On the top of the base model, a set of layers including pooling, fully connected and dropout were added to create the classifier. The description of these layers has been given below.

\section{Average Pooling2D Layer}

The AveragePooling2D layer receives the input from the stage 1. The main aim of using average pooling layer is to progressively reduce the spatial size of the input, number of parameters and 
computation in the network. The filter size of $2 \times 2$ has been used. Subsequently output of the average pooling layer is flattened to give a $1 \mathrm{D}$ array which serves as an input for the next layer.

\section{Fully Connected Layer}

Fully Connected (FC) layers are similar to typical ANN layers. The FC layer takes input from the previous layer, passes the linear calculated function of inputs through an activation function to give the output for that node. The output serves as input for the next layer. For FC layer, 128 nodes have been used and the function used is Re-Lu activation as it helps in learning more complex features by adding non-linearity in the network.

\section{Dropout}

Subsequently, dropout of 0.5 is used. This means for the next layer, only half of the randomly selected neurons will be selected for input. Dropout is an approach to regularization in neural networks which helps reducing interdependent learning amongst the neurons. This helps us to prevent over fitting,

\section{Output Layer}

The last layer in our model is a Dense Layer or Fully Connected Layer with only 3 nodes (for 3 classes). This layer is a softmax layer which uses the softmax activation function. This layer is used to derive the output of the model.

\section{Implementation Details}

\subsection{Dataset}

The dataset used in this paper has also been used in DarkCovidNet Model study[14]. The dataset is combination of two datasets which are:

1. The database prepared by Cohen JP[20]

2. ChestXRay-8 dataset prepared by Wang et al[21].

Covid-19 chest X-ray images have been extracted from the database prepared by Cohen JP[20] while Normal and Pneumonia chest X-ray images have been extracted from dataset prepared by Wang et al[21].For training set , 162 Covid-19 X-ray images and 225 images each of Pneumonia and Normal class selected randomly from the above mentioned datasets were used. For validation set, 18 Covid-19 X-ray images and 25 images each of Pneumonia and Normal class selected randomly from the abovementioned datasets were used. For test set, 30 images each of Covid-19, Pneumonia and Normal class were used. Hence, a total of 612 images have been used for training set ,68 images for validation set and 90 images have been used for testing purpose. All the training images are different from testing images. All the images available for pneumonia and normal classes in the 2 datasets weren't used so as to prevent class imbalance problem.

\subsection{Environment Setup}

Dataset was prepared on a local computer with Intel(R) Core (TM) i7-2.2 GHz processor with 16 GB RAM. Dataset was subsequently uploaded on Google drive. Experiments were carried out on Google Colab and graphical processing unit provided by Colab was used.

\subsection{Training Hyperparameters}

Table 2 gives various hyperparameters used in the paper. 
Table 2. Training Hyperparameters

\begin{tabular}{ll}
\hline Hyperparameter Name & Value \\
\hline Train-Validation-Test split ratio & $79 \%-9 \%-12 \%$ \\
Batch Size & 32 \\
Number of Epochs & 100 \\
Learning Rate & $5 \mathrm{e}-5$ \\
Data Scaling Size & $224 \times 224$ \\
\hline
\end{tabular}

Apart from these, the overfitting was kept in check by applying callback once training accuracy reaches $99 \%$ and applying a dropout of 0.5 .

\subsection{Performance Parameters}

Confusion matrices have been plotted which gives us the values of True Positives (TP), False Positives (FP), False Negatives (FN) and True Negatives (TN). Based on these, parameters and metrics used in this study to measure performance of the classifiers are:

\section{Accuracy}

Accuracy of a classifier is defined as sum of total positives and total negatives divided by total number of samples. Accuracy is one of the most important metrics to assess the performance of the deep learning classifier.

$$
\begin{aligned}
& \operatorname{Accuracy}(\%) \\
& =\frac{(T P+T N)}{(T P+T N+F P+F N)} * 100 \%
\end{aligned}
$$

\section{Sensitivity or recall}

Sensitivity is defined as true positives divided by sum of True Positives and False Negatives. It is advised to have a high sensitivity in medical field-oriented classifier as a person who is infected with a disease, if is predicted negative (False Negatives), then he could spread the disease to more people. To increase the sensitivity, False Negatives should be lowered.

$$
\text { Sensitivity }=\frac{T P}{T P+F N}
$$

\section{Specificity}

Specificity is defined as number of true negatives divided by total predicted negatives.

$$
\text { Specificity }=\frac{T N}{T N+F N}
$$

\section{Results and Analysis}

After training the classifiers, their performance was analyzed on the basis of metrics mentioned in the previous section. Figure 2 below shows all the confusion matrices obtained for different networks on the test set. Through confusion matrices, we're able to obtain values of True Positives (TP), False Positives (FP), False Negatives (FN) and 
True Negatives (TN). Though most of the classifiers have almost similar number of $\mathrm{TN}+\mathrm{TP}$, Inception ResNetV2 has highest total of TN+TP. Inception ResNetV2 and Xception have $100 \%$ accuracy in predicting the Covid-19 class.

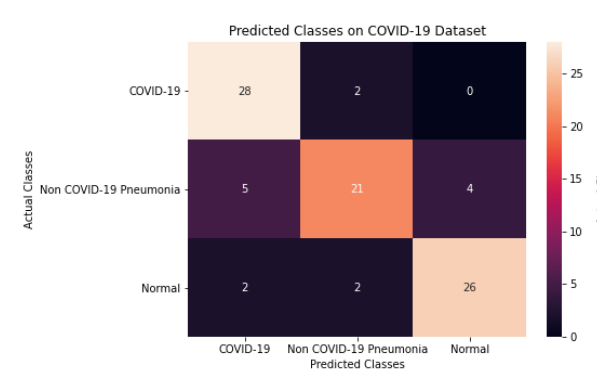

(a) DenseNet201

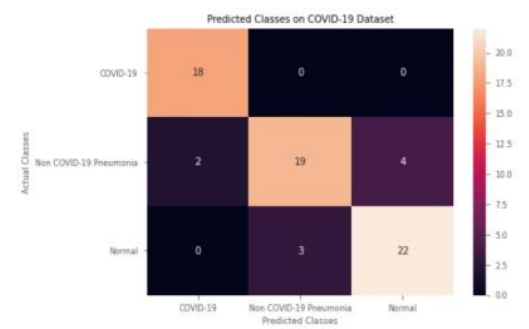

(c) Xception

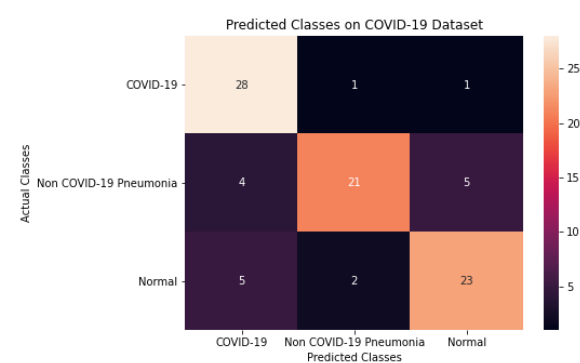

(e ) VGG16

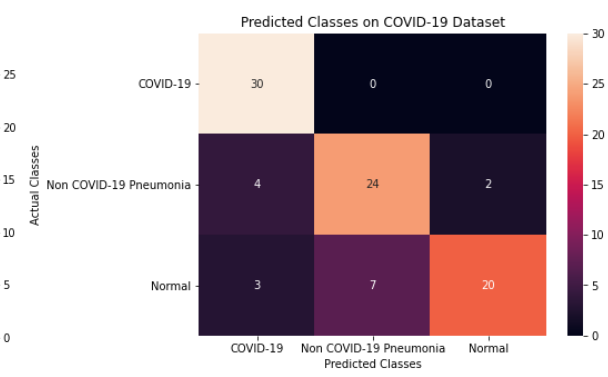

(b)InceptionResNetV2

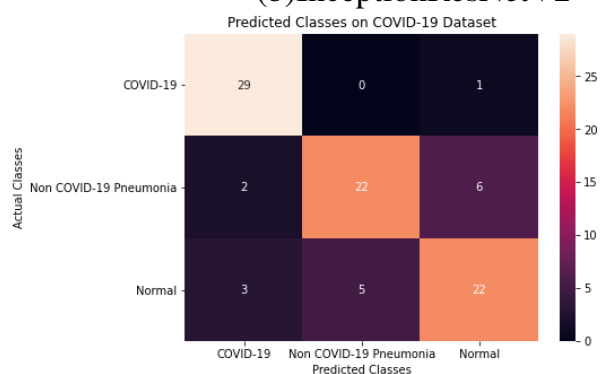

(d)ResNet50V2

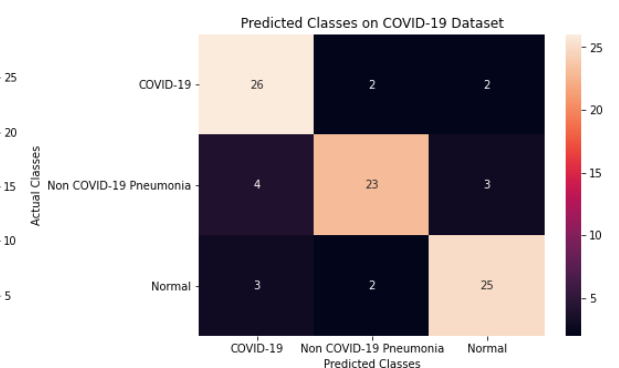

(f) VGG19

Fig 2: Confusion Matrix for each of the deep learning networks

Table 3 gives comparative study of the accuracy, sensitivity and specificity of all the classifiers based on the confusion matrices in Figure 2. Table 4 gives precision, recall and F1-score values for each of the 3 classes and each of the classifiers. Highest accuracy of $83.33 \%$ was achieved by Inception ResNetV2. However, other networks like DenseNet201, VGG19 and Xception also achieved a comparably similar accuracy of $82.22 \%$. Lowest accuracy of $81.11 \%$ was obtained by VGG16. DenseNet201 and Xception attain the highest sensitivity of 1.00 while highest specificity of 0.80 was achieved by VGG 19 . Lowest sensitivity of 0.80 was obtained by VGG 19 High sensitivity is desirable in medical field-oriented classifier as a person who is infected with a disease, if is predicted negative (False Negatives), then he could spread the disease to more 
people. DenseNet201 and Xception perform better than the other classifiers on this aspect while VGG-19 performs worse than the other classifiers.

For detection of only Covid-19 class, all three classifiers, Inception ResNetV2, DenseNet201 and Xception attained almost the same precision value. For the rest of two classes, InceptionResNetV2 has high precision values for both normal and pneumonia class while DenseNet201 has high precision for normal class but low precision for pneumonia class. DenseNet201 and Xception attain a maximum possible recall of 1.00 for Covid-19 class while DenseNet201 has highest recall of 0.80 for Pneumonia class and InceptionResNetV2 had highest recall of 0.87 for Normal class. Also, DenseNet 201 and Xception achieved the highest F1-score of 0.90 for Covid-19 class which is desirable for the results. So, for this classification task, InceptionResNetv2, Xception and DenseNet201 achieve almost similar results.

Table 3. Accuracy, Sensitivity and Specificity for each of deep learning networks

\begin{tabular}{llcc}
\hline Classifier & Accuracy $(\%)$ & Sensitivity & Specificity \\
\hline Xception & 82.22 & 1.00 & 0.73 \\
VGG16 & 80.00 & 0.93 & 0.73 \\
VGG19 & 82.22 & 0.87 & 0.80 \\
DenseNet2 & 82.22 & 1.00 & 0.73 \\
01 & & 0.93 & 0.78 \\
InceptionR & 83.33 & 0.97 & 0.73 \\
esNetV2 & & & \\
ResNet50V & 81.11 & & \\
\hline
\end{tabular}

Table 4. Precision, Recall and F1-score values for each of the 3 classes and deep learning networks.

\begin{tabular}{lllll}
\hline Heading level & Class & Precision & Recall & F1 Score \\
\hline Xception & Covid-19 & 0.81 & 1.00 & 0.90 \\
& Pneumonia & 0.81 & 0.73 & 0.77 \\
VGG19 & Normal & 0.85 & 0.73 & 0.79 \\
& Covid-19 & 0.79 & 0.87 & 0.83 \\
VGG16 & Pneumonia & 0.85 & 0.77 & 0.81 \\
& Normal & 0.83 & 0.83 & 0.83 \\
& Covid-19 & 0.76 & 0.90 & 0.84 \\
DenseNet201 & Pneumonia & 0.88 & 0.73 & 0.78 \\
& Normal & 0.79 & 0.77 & 0.78 \\
InceptionResNetV & Covid-19 & 0.81 & 1.00 & 0.90 \\
2 & Pneumonia & 0.77 & 0.80 & 0.79 \\
& Normal & 0.91 & 0.67 & 0.77 \\
ResNet50V2 & Covid-19 & 0.80 & 0.93 & 0.86 \\
& Pneumonia & 0.84 & 0.70 & 0.77 \\
& Normal & 0.87 & 0.87 & 0.87 \\
& Covid-19 & 0.85 & 0.97 & 0.91 \\
& Pneumonia & 0.81 & 0.73 & 0.77 \\
\hline
\end{tabular}


Figure 3 gives the accuracy and loss curves for each of the classifiers. Train loss, validation loss, train accuracy and validation accuracy have been plotted on those graphs. From the plots, we could see that the plots of VGG16 and VGG 19 are not smooth. While analyzing the loss and accuracy graphs from figure 3, it is observed that graph for DenseNet201 is smoother and finer as compared to the graphs of InceptionResNetV2 and Xception. Also, DenseNet201 took around just 77 epochs to train after which callback was used as compared to 100 steps for both InceptionResNetV2 and Xception networks.

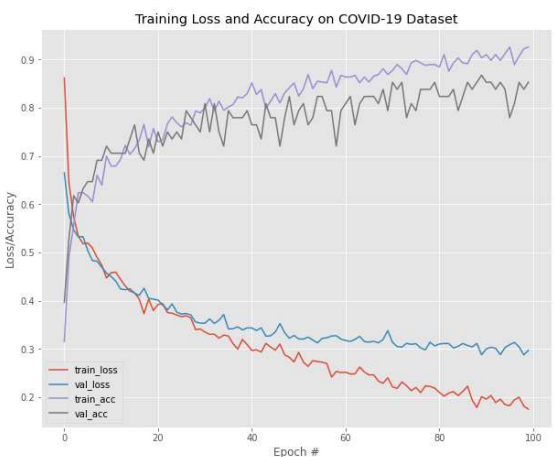

(a) InceptionResNetV2

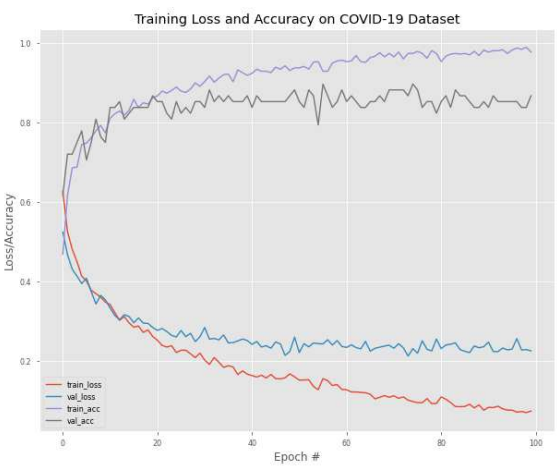

(c) Xception

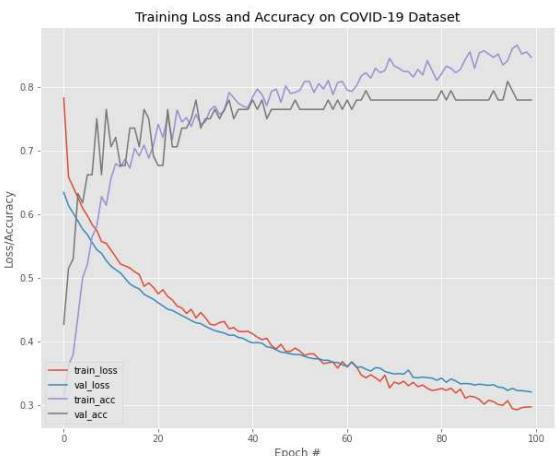

(e) VGG16

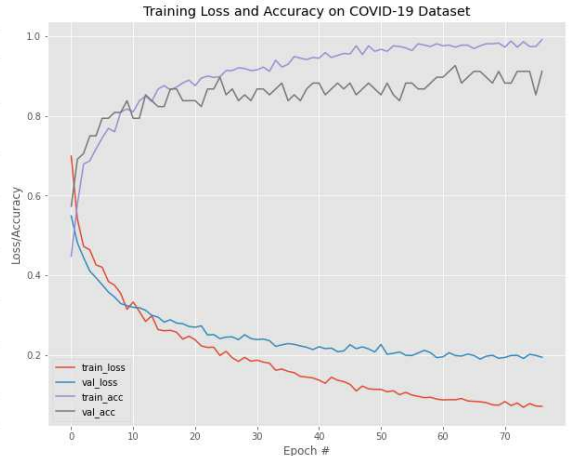

(b) DenseNet201

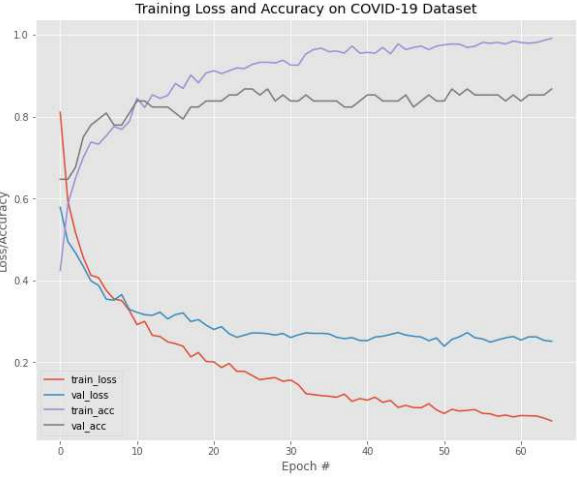

(d )ResNet50V2

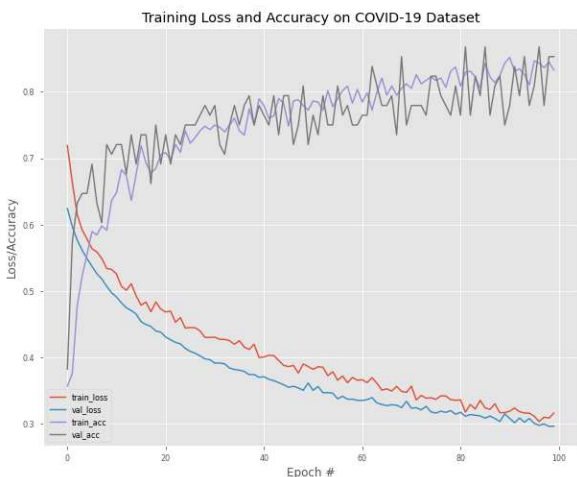

(f) VGG19

Fig 3: Loss and Accuracy curves for each of the deep learning networks 
So, after analyzing the above results, we could infer that InceptionResNetv2, Xception and DenseNet201 have almost similar performance based on the performance parameters. However, after analyzing the loss and accuracy curves, it could be inferred that the training phase of DenseNet201 was both faster and smoother than Xception and InceptionResNetV2.

Hence, DenseNet201 network is recommended for our proposed model.

\section{Conclusion and Future Work}

In 2020, Covid-19 brought the whole world to a standstill. Millions of people died due to this deadly virus. Less efficiency and higher diagnosing time of test kits and traditional methods of detection required a substitute. Hence, in this study, transfer learning has been used to make a deep learning architecture to perform multi-class classification between the 3 classes (Covid-19, Pneumonia, Normal) using Chest X-Ray images. The study is based on 6 different pre-trained networks. The proposed model in the study was analysed based on different deep learning networks and their performances were compared on basis of different performance parameters and it was found that three classifiers, IncetionResNetV2, Xception and DenseNet201 attained similar metrics. But on further scrutiny, DenseNet201 was found to have an edge over the other two, hence DenseNet201 is recommended network for our proposed model. In the future, this study can be extended to multi-modal classification by examining the effects of the disease on different organs of the body, and not just the lungs. Multi-Modal classification may also be done in future by using and incorporating different types of radio-logical images available such as CTscan. Also, the study can be extended to classification of bacterial and viral pneumonia along with Covid-19 and normal class.

\section{References}

[1] “covid-19 - Google Search.” https://www.google.com/search?q=covid$19 \& \mathrm{rlz}=1 \mathrm{C} 1 \mathrm{RLNS} \_$enIN880IN881\&oq=covid19+\&aqs=chrome..69i57j0i131i43313j0i131i433i457j69i61j69i6012.5451j0j7\&sourcei $\mathrm{d}=$ chrome\&ie=UTF-8 (accessed Mar. 30, 2021).

[2] "Coronaviruses - National Foundation for Infectious Diseases." https://www.nfid.org/infectious-diseases/coronaviruses/ (accessed Mar. 30, 2021).

[3] "Symptoms of Coronavirus: Early Signs, Serious Symptoms and More." https://www.webmd.com/lung/covid-19-symptoms\#1 (accessed Mar. 30, 2021).

[4] "Coronavirus: Diagnosis and Testing of COVID-19." https://www.healthline.com/health/coronavirus-diagnosis\#when-to-get-a-diagnosis (accessed Mar. 30, 2021).

[5] S. Francis, R. P. Mathew, and Z. A. Khalid, "Coronavirus (COVID-19) Infection in Pregnancy: Does Non-contrast Chest Computed Tomography (CT) Have a Role in Its Evaluation and Management?," J. Obstet. Gynecol. India, vol. 70, no. 4, pp. 272-274, 2020, doi: 10.1007/s13224-020-01341-5.

[6] E. E. D. Hemdan, M. A. Shouman, and M. E. Karar, "COVIDX-Net: A Framework of Deep Learning Classifiers to Diagnose COVID-19 in X-Ray Images," arXiv, 2020.

[7] J. Lee et al., "2017-Lee-Deep Learning in Medical Imaging_Gen," vol. 18, no. 4, pp. 570-584, 2017.

[8] R. H. Abiyev and M. K. S. Ma'aitah, "Deep Convolutional Neural Networks for Chest Diseases Detection," J. Healthc. Eng., vol. 2018, p. 4168538, 2018, doi: $10.1155 / 2018 / 4168538$.

[9] "Transfer Learning in Keras with Computer Vision Models." 
https://machinelearningmastery.com/how-to-use-transfer-learning-when-developingconvolutional-neural-network-models/ (accessed Mar. 30, 2021).

[10] Antonios Makris, I. Kontopoulos, and K. Tserpes, "COVID-19 detection from chest $\mathrm{X}$-Ray images using Deep Learning and Convolutional Neural Networks," Sensors, vol. 21, no. 4, pp. 1-30, 2021, doi: 10.3390/s21041480.

[11] H. Gunraj, L. Wang, and A. Wong, "COVIDNet-CT: A Tailored Deep Convolutional Neural Network Design for Detection of COVID-19 Cases From Chest CT Images," Front. Med., vol. 7, pp. 1-12, 2020, doi: 10.3389/fmed.2020.608525.

[12] I. Castiglioni et al., "Artificial intelligence applied on chest X-ray can aid in the diagnosis of COVID-19 infection: a first experience from Lombardy, Italy." medRxiv, 2020, doi: 10.1101/2020.04.08.20040907.

[13] A. Narin, C. Kaya, and Z. Pamuk, "Department of Biomedical Engineering, Zonguldak Bulent Ecevit University, 67100, Zonguldak, Turkey.," arXiv Prepr. arXiv2003.10849., 2020, [Online]. Available: https://arxiv.org/abs/2003.10849.

[14] T. Ozturk, M. Talo, E. A. Yildirim, U. B. Baloglu, O. Yildirim, and U. Rajendra Acharya, "Automated detection of COVID-19 cases using deep neural networks with X-ray images.," Comput. Biol. Med., vol. 121, p. 103792, Jun. 2020, doi: 10.1016/j.compbiomed.2020.103792.

[15] K. Simonyan and A. Zisserman, "Very deep convolutional networks for large-scale image recognition," 3rd Int. Conf. Learn. Represent. ICLR 2015 - Conf. Track Proc., pp. $1-14,2015$

[16] G. Huang, Z. Liu, L. Van Der Maaten, and K. Q. Weinberger, "Densely connected convolutional networks," Proc. - 30th IEEE Conf. Comput. Vis. Pattern Recognition, CVPR 2017, vol. 2017-January, pp. 2261-2269, 2017, doi: 10.1109/CVPR.2017.243.

[17] F. Chollet, "Xception: Deep learning with depthwise separable convolutions," Proc. 30th IEEE Conf. Comput. Vis. Pattern Recognition, CVPR 2017, vol. 2017-January, pp. 1800-1807, 2017, doi: 10.1109/CVPR.2017.195.

[18] C. Szegedy, S. Ioffe, V. Vanhoucke, and A. A. Alemi, "Inception-v4, inceptionResNet and the impact of residual connections on learning," 31st AAAI Conf. Artif. Intell. AAAI 2017, pp. 4278-4284, 2017.

[19] K. He, X. Zhang, S. Ren, and J. Sun, "Deep residual learning for image recognition," Proc. IEEE Comput. Soc. Conf. Comput. Vis. Pattern Recognit., vol. 2016-December, pp. 770-778, 2016, doi: 10.1109/CVPR.2016.90.

[20] J. Paul Cohen, P. Morrison, and L. Dao, "COVID-19 Image Data Collection," arXiv, 2020.

[21] X. Wang, Y. Peng, L. Lu, Z. Lu, M. Bagheri, and R. M. Summers, "ChestX-ray: Hospital-Scale Chest X-ray Database and Benchmarks on Weakly Supervised Classification and Localization of Common Thorax Diseases," Adv. Comput. Vis. Pattern Recognit., pp. 369-392, 2019, doi: 10.1007/978-3-030-13969-8_18. 